\title{
Review of: "Computational Modeling of Chromatin Accessibility Identified Important Epigenomic Regulators"
}

\author{
Fang $\mathrm{Li}^{1}$ \\ 1 Tongji University
}

Potential competing interests: The author(s) declared that no potential competing interests exist.

Zhao et al proposed a computational framework of chromatin accessibility prediction by using histone modifications (HMs) and transcription factors (TFs). Authors well predicted global manner chromatin accessibility in GM12878 and HepG2 cell lines and thus they inferred that HMs or TFs might enable to regulate chromatin accessibility. They further identified several chromatin accessibility-associated HMs and histone variants, especially H2AZ.1. The also verified combining HMs and TFs did not improve the accuracy of chromatin accessibility. These findings enlarged our view in chromatin accessibility regulation and gave a novel idea that TFs could regulate chromatin accessibility. However, some concerns remain, and the author need to reply:

Minor revise:

1. The application of these prediction model needs to be elaborated clearly. For samples from both cell lines and tissues samples, the ATAC-seq is the better choice for exploring chromatin accessibility economically and technically. Why should we predict the results of chromatin accessibility by using ChIP-seq in the case of that at least one ChIP-seq data should be acquired first? In general, we conduct ATAC-seq to identify which TFs is significant in this cell line or tissue sample and then verify our findings by ATAC-seq.

2. The authors selected several HMs or TFs could predict global manner chromatin accessibility across cell lines. Whether it is available to confirm this point in more cell lines collected by ENCODE?

3. It is well known that HMs could serve as marker of specific location of TSS, promoter or enhancer, but what is the possible mechanism of association between TFs and chromatin accessibility? Whether it is possible that those TFs could predict chromatin accessibility are general TFs which motifs widely distribute in genome? Please cite more references to illustrate it. 